\title{
Prognostic and Diagnostic Value of Clinical Examination and fMRI in the Evaluation of Patients in a Vegetative State
}

Damiani Sabino ${ }^{1}$, Marvulli Riccardo ${ }^{1 *}$, Dargenio Maria ${ }^{1}$, Gallo Giulia Alessia1, Salvati Andrea ${ }^{2}$, Fiore Pietro ${ }^{1}$ and Megna Marisa ${ }^{1}$

${ }^{1}$ Department of Basic Sciences, Neuroscience and Sense Organs, University of Bari "Aldo Moro", Bari, Italy

${ }^{2}$ General Hospital "F. Miulli", O.U. Radiology, Acquaviva delle Fonti (Bari), Italy

\begin{abstract}
Introduction: Primary focus of research in psychology, neuropsychology and neuroscience within the past few decades understands what consciousness means biologically and psychologically for information present in it that is on determining the neural and psychological correlates of consciousness. This retrospective study evaluated the correlation between clinical examination and neuroradiological imaging in patients with disorder of consciousness (DOC), for predicting the likely outcome of one's current standing
\end{abstract}

Materials and methods: Six patients (4 men and 2 female) suffered of DOC post-coma, outcome of severe acquired brain injuries were examined in acute phase with brain (scan) CAT (computerized axial tomography) and/ or fMRI (functional magnetic resonance imaging). After, they were evaluated with electroencephalogram and fMRI. Repeated clinical assessments were conducted with neurological examination and rating scales such as Glasgow Coma Scale (GCS) and Disability Rating Scale (DRS) at the beginning (T1), middle (T2) and end (T3) of hospitalization. $2 / 6$ patients died.

Discussion: Clinical evaluation performed with rating scales of consciousness and disability degree, showed in all patients an increase of absolute value of GCS and a reduction of DRS. We obtained most interesting observations concerning the relationship between the prognosis and patient characteristics with functional MRI.

Conclusion: Although the few numbers of patients and the limits of methodological problems, our study, in accordance with previous evidence from the literature, may confirm the central role of nature of brain injuries as the prediction marker for the and fMRI direct visualization of the areas of cortical activation.

Keywords: Disorder of consciousness; fMRI; Cortical activation; Brain injuries; Functional connectivity

\section{Introduction}

According to recent developments in technology, consciousness has become a significant topic of research in psychology, neuropsychology and neuroscience within the past few decades. Primary focus understands what it means biologically and psychologically for information present in consciousness that is on determining the neural and psychological correlates of consciousness [1]. Perhaps, consciousness and our conscious experience of self is more familiar or more puzzling world aspect of mind. We need to understand both what consciousness is and how it relates to other non-consciousness aspects of reality. Consciousness allows us to have an experience; without it we can have no experiences of all. Applying measures of neural integration and complexity and analysis of extensive neurological data, leads to a testable proposal about the properties of the neural substrate of consciousness (dynamic core hypothesis) [2].

In the 60's, Plum and Posner [3], Crick and Koch [4] and other researchers were evaluated and restored cognitive functions and consciousness in patients with a disorder of consciousness (DOC) following a severe brain injuries. They undertook a series of studies and identified a "general theory of consciousness" and "theory of the neural correlates of consciousness". Disorders of consciousness include patient in a persistent vegetative state and in a minimally conscious state. However, multiple studies demonstrated that clinical diagnoses of these conditions are inaccurate, even when improved through the use of standardized scoring systems such as the Coma Recovery Scale-Revised (CRS-R). Different imaging and electrophysiological techniques have recently been reported to improve the accuracy of these diagnoses; a recent study of Stender examined two different neuroimaging techniques in a relatively large and well-characterized cohort of patients. The authors examined FDG-PET as well as functional MRI (fMRI) in patients with disorders of consciousness. It has been hypothesized that PET can distinguish between patients in a persistent vegetative state and those in a minimally conscious state based on specific areas of hypo metabolism in the brain. Using fMRI in patients asking to perform mental tasks such as motor imagery, clinicians may be able to determine patients who have some preserved level of consciousness even when there are no outward signs of such [5].

According to the information integration theory, consciousness corresponds to the capacity of a system to integrate information [6] This claim is motivated by two key phenomenological properties of consciousness: differentiation (the availability of a very large number of conscious experiences) and integration (the unity of each such experience). Several neurobiological observations include the association of consciousness with certain neural systems rather than with others, such as neural processes underlying consciousness can influence or be influenced by neural processes that remain unconscious and the reduction of consciousness during dreamless sleep and

*Corresponding author: Marvulli Riccardo, Department of Basic Sciences Neuroscience and Sense Organs, University of Bari "Aldo Moro", Bari, Italy, Tel: +390805593499; Fax: 0805478580; E-mail: ricmarv81@hotmail.it

Received May 10, 2017; Accepted June 22, 2017; Published June 29, 2017

Citation: Sabino D, Riccardo M, Maria D, Alessia GG, Andrea S, et al. (2017) Prognostic and Diagnostic Value of Clinical Examination and fMRI in the Evaluation of Patients in a Vegetative State. J Neurol Neurophysiol 8: 430. doi:10.4172/2155 9562.1000430

Copyright: $\odot 2017$ Sabino D, et al. This is an open-access article distributed under the terms of the Creative Commons Attribution License, which permits unrestricted use, distribution, and reproduction in any medium, provided the original author and source are credited. 
generalized seizures. Consciousness is generated by the thalamocortical system that integrates information among distributed cortical regions (reduced in sleep and epilepsy).

The ascending reticular activating system (ARAS) mediates arousal, an essential component of human consciousness. Studies revealed novel human pathways connecting the brainstem to the thalamus, the hypothalamus (important in DOC) and the basal forebrain. Thus, the waveforms of the cortical EEG appear to be due to complex interactions among the burst neurons in the thalamus, cortex and basal forebrain, all of which receive substantial inputs from the ascending arousal system.

The epidemiologic pattern of traumatic brain injuries (TBI), one of the most important causes of death in patients under 25 years old and responsible for one third of total deaths caused by trauma, is increasing in emergency department [7]. In addition, there have been significant advances in neurosurgery and reanimation [8]. DOC is thus not just a health problem. It is a complex phenomenon, reflecting the interaction between features of a person's body and features of the society in which he or she lives.

Those patients typically develop dependence in toileting, feeding and dressing and participate in work environments. Patients' evaluation with DOC requires a history, physical examination and laboratory evaluation. DOC patient's present problems related to the ongoing risks of immobility and artificial respiration and feeding, including pneumonia, urinary tract infection and pressure ulcers. Others were related to ongoing evolution of the neurological injuries, including development or worsening of hypertonia and contractures and emergence of post-traumatic agitation. Cause frequent medical complications, rehabilitation programs need the capacity to diagnose these problems early to employ a systematic approach to medical management [9].

The nursing care of patients with DOC must be particular and specific according to difficult diagnosis, problem of unconsciousness or lack of demonstration of consciousness and extremely complex clinical assessment.

DOC includes patients in coma, in vegetative/unresponsive wakefulness syndrome (VS/UWS) and in minimally conscious state (MCS). Patients in VS/UWS are characterized by the presence of reflexive responses to external stimuli and are considered unconscious. Patients in MCS show reproducible but minimal and fluctuating signs of consciousness. By definition, these patients are unable to express their feelings and cannot communicate about potential discomfort or pain.

Plum and Posner conceived coma to be total absence of awareness of self and environment even when the subject is externally stimulated [10]. Coma is defined as a state of un-arousable (impairment of arousable), unresponsiveness (impairment of cognitive content) in which the subject lies with their eyes closed, no spontaneous eye opening, no response to voice and no localization to painful stimuli or verbal output.

VS denotes the recovery of crude cycling of arousal states heralded by the appearance of "eyes-open" periods in an unresponsive patient. Very few surviving patients with severe forebrain damage remain in eyes-closed coma for more than 10 to 30 days. In most patients, vegetative behavior usually replaces coma by that time. Patients in the vegetative state, like comatose patients, show no evidence of awareness of self or their environment [11]. A patient in vegetative state is unconscious but his eyes are opened, they can breathe on his or her own (but requires artificial feeding), automatic-vegetative functions and some active functions of the severely damaged brain are preserved.

Although the original term persistent vegetative state (PVS) was not associated with a specific time, the use of PVS is now commonly reserved for patients remaining in a vegetative state for at least 30 days. Vegetative state may be regarded as permanent 3 months after nontraumatic brain damage or 12 months after traumatic injuries.

The minimally conscious state (MCS) is defined by the presence of inconsistent but reproducible goal-directed behaviors (e.g. response to command, verbalizations, visual pursuit, etc.) [12]. Like the vegetative state, MCS often exists as a transitional state arising during recovery from coma or worsening of progressive neurologic disease.

MCS was recently subcategorized based on the complexity of patients' behaviors: MCS+ describes high-level behavioral responses (i.e., command following, intelligible verbalizations or non-functional communication) and MCS- describes low-level behavioral responses (i.e., visual pursuit, localization of noxious stimulation or contingent behavior such as appropriate smiling or crying to emotional stimuli) [13].

Behavioral assessment remains the "gold standard" for detecting signs of consciousness and, hence, for determining diagnosis [14].

However, behavioral assessment is complicated by the presence of motor impairment, tracheotomy, fluctuating arousal level or ambiguous and rapidly habituating responses [15].

Previous studies showed $37-43 \%$ of patients diagnosed with VS demonstrated signs of awareness [16,17].

Misdiagnosis can lead to grave consequences, especially in end-oflife decision-making [18].

Contrary to patients in VS, those in MCS retain some capacity for cognitive processing and activate similar brain networks relatives to controls following painful stimulation, suggesting that they can experience pain $[19,20]$.

Differentiating VS from MCS is often one of the most challenging tasks facing clinicians involved in the care of patients with disorders of consciousness (DOC) [21].

Patients in MCS will show more than the mere reflex behavior observed in VS survivors, but they are unable to effectively communicate. Preliminary evidence indicates that MCS patients attain better functional improvement and demonstrate improvement over a longer period of time as compared to those in VS [22,23].

It is much more difficult to predict the outcome for DOC patients. Therefore, it is very important to determine the level of consciousness. The accurate diagnosis and proper cognitive assessment are critical for the rehabilitation of patients with disorders of consciousness.

\section{Neuroimaging in patients with DOC}

Functional magnetic resonance imaging (fMRI) is a relatively new procedure that uses MR imaging to measure the tiny metabolic changes that take place in an active part of the brain (when a brain area is more active, it consumes more oxygen and this increases demand blood flow). It allows us the detection of structural, functional and metabolic changes useful not only for differential diagnosis, but also for early diagnosis and outcome and treatment monitoring in DOC patients.

Functional MRI and PET (which traces changes in glucose 
metabolism on a slow time scale) define the brain function in response to a stimulus.

Hemoglobin is diamagnetic when oxygenated but paramagnetic when deoxygenated. This difference in magnetic properties leads to small differences in the MR signal of blood depending on the degree of oxygenation. Since blood oxygenation varies according to the levels of neural activity, these differences can be used to detect brain activity. This form of MRI is known as blood oxygenation level dependent (BOLD) imaging.

The data provided by these methods can be used to predict longterm neurological outcomes. But only the conventional MRI fails to accurately predict outcomes.

These methods allow us to observe the brain activity in response to sensory stimuli (acoustic, visual, tactile) during the execution of tasks or during a resting state.

PET shows a functional recovery of metabolism of the vegetative state in the brainstem (encompassing the reticular formation, the hypothalamus and the basal forebrain) [24]. This allows for the maintenance functions in these patients such as sleep wake cycles, autonomic and ventilatory control and cranial nerve reflexed.

In VS patients the resumption of long-range functional connectivity between the fronto-parietal cortices and between some of these and the intra-laminar thalamic nuclei parallels the restoration of their functional integrity. The importance of networking is particularly high in some functions of consciousness like speech, attention, and memory.

A common finding of studies on pathological coma is impairment in the activity of a widespread cortical network, encompassing bilateral fronto-parietal associative cortices and mid-cingulate area [25,26]. These structures are the most active in the resting not stimulated, defined Brain Default Network state (BDN), network of interacting brain regions known to have activity highly correlated with each other and distinct from other networks in the brain [27].

The same functional impairment frontal parietal is seen in other cases of unconsciousness such as sleep, coma, general anesthesia, generalized epilepsy, states of absence, complex partial epilepsy and somnambulism [28-31].

Recently, connectivity in all default network areas was found to be linearly correlated with the degree of consciousness, ranging from healthy volunteers and locked-in syndrome to minimally conscious, vegetative, and comatose patients. Furthermore, precuneus connectivity was found to be significantly stronger in minimally conscious patients compared to vegetative-state patients. Locked-in syndrome patients' default network connectivity was shown not to be significantly different from healthy control subjects.

DOC patients represent a clinical problem, in terms of diagnosis, prognosis, treatment and everyday management. In fact, these patients represent genuine cases of abolition of consciousness with preserved arousal, contrary to coma patients. Consciousness is thought to represent an emergent property of cortical and subcortical neural networks and their reciprocal projections. Its multifaceted aspects can be seen as expressions of various specialized areas of the cortex that are responsible for processing external and internal stimuli, shortand long-term storage, language comprehension and production, information integration and problem solving and attention.

Recently a large body of work improved our knowledge about possible residual cognitive functioning of DOC patients. In particular, neurophysiologic and functional brain imaging studies consistently showed that a subset of DOC patients are able to produce some covert responses (e.g. hand movements), despite the lack of any overt behavioral manifestation, suggesting a preservation of islands of highorder cognitive functioning (e.g. speech processing, mental imagery, etc.).

The relevant fact emerged in these studies is that somatosensory stimulation of PVS patients, at intensities that elicited pain in controls, resulted in increased neuronal activity in primary somatosensory cortex, even if resting brain metabolism was severely impaired. However, this activation of primary cortex seems to be isolated and dissociated from higher-order associative cortices.

It is investigated somatosensory processing in PVS patients, using high-intensity electrical stimulation of the median nerve at the wrist. In controls, stimulation resulted in the subjective experience of pain and increased response in midbrain, contralateral thalamus and contralateral primary somatosensory, contralateral secondary somatosensory, bilateral insular, posterior parietal and anterior cingulate cortices. In patients, stimulation increased regional neural activity in brain stem and contralateral thalamus and contralateral primary somatosensory.

A study describes that in controls, auditory stimulation activated auditory cortex contralateral and ipsilateral to the side of stimulation [32]. The activated areas encompassed bilateral transverse temporal gyrus (TTG) and superior temporal gyrus (STG). In patients in an MCS, auditory stimulation activated bilateral auditory cortex. In patients in a PVS, auditory stimuli also activated the bilateral auditory cortex, but the extent of this activation was much smaller. In response to auditory stimulation, patients in an MCS activated spatially larger areas of auditory cortex than did patients in a PVS. Functional connectivity between the secondary auditory cortex and the posterior temporal and prefrontal areas, involved in higher levels of auditory processing, was significantly more effective in patients in an MCS than in patients in a PVS. In agreement with our previous results, controls activated bilateral temporal areas. Patients in a PVS activated bilateral temporal areas, but not higher-order associative area. Nevertheless, the activation of higher-order associative temporal cortices found in patients in an MCS probably corresponds to a more elaborate auditory processing, allowing further cognitive integration of the stimuli [32].

Visual stimuli typically activate primary cortices in patients with VS and fail to show robust activation in higher order associative cortices. Positron emission tomography activation studies suggest that the vegetative state represents a global disconnection syndrome in which higher order association cortices are functionally disconnected from primary cortical areas. In contrast, patterns of activation in functional magnetic resonance imaging studies of patients in the minimally conscious state show preservation of large-scale cortical networks associated with language and visual processing [33,34].

Some VS patients showed "higher level" associative activation. This type of activation patterns has been called "atypical". Neuroimaging data seem to show that atypical 'higher order' associative cortical activation in VS herald recovery of some level of consciousness some months later. The analysis of functional neuroimaging data published on VS patients shows that a high level associative cortical activation (as compared to absent or low-level primary activation) seems to predict recovery of consciousness with $93 \%$ specificity and $69 \%$ sensitivity. It is observed the activation in anterior cingulate, right middle temporal and right premotor areas when the patient was presented a story told by the patient's mother (as compared to non-word sounds). Furthermore, 
compared to other names, SON activated bilateral medial prefrontal, left temporal-parietal and superior frontal cortices.

The left inferior frontal gyrus plays a vital role in the combinatorial aspects of sentence comprehension. Recent fMRI studies have consistently highlighted the role of the left inferior frontal gyrus in resolving semantic ambiguities within sentences compared spoken sentences that contained several ambiguous words (e.g. the shell was fired toward the tank) with well-matched low-ambiguity sentences (e.g. her secrets were written in her diary). This semantic ambiguity response is sufficiently robust that it has been used as a marker of preserved speech processing in patients with disorders of consciousness [35].

Due to the complexity of DOC, outcome measurement is a multifaceted issue. It is difficult to determine the cause of lack of response in DOC patients. In fact, it can depend on impairment of arousal or aphasia, akinesia or motor disorders.

Therefore, if fMRI shows a significant activation of the temporal structures involved in language processing, but a minimal activation of the mesial frontal structures involved in movement, it would be reasonable to assume that akinesia plays a principal role in the failure to respond to commands.

Consequently, the rehabilitation program will include the use of neurostimulation rather than therapy for aphasia.

\section{Pharmacological agents in patients with DOC}

Georgiopoulos et al. recently reviewed therapeutic intervention in chronic DOC (over 6 months in posttraumatic cases and over 3 months in nontraumatic cases) [36]. Results showed utility of dopaminergic agents in DOC: Levodopa and Amantadine both in SV and MCS to produce a clinical improvement within 4 days to 1,5 months. Authors defined recovery patient's ability to communicate and to obey simple commands or functional object use. It has been suggested that levodopa can be useful because of the interruption of the vicious circle of exhaustion of neurotransmitters. Levodopa inefficacy does not predict other dopaminergic agents' efficacy because of different mechanism of action. Amantadine acts both pre- and post- synaptic sites causing an increase of dopamine release through post-synaptic facilitation.

Both tricyclic antidepressant (TCAs), such as protriptyline, amitriptyline and desipramine and selective reuptake inhibitors (SSRIs) such as sertraline, can improve arousal or initiation in vegetative patients following severe brain injuries [37].

Methylphenidate, a pre-synaptic catecholamine mimetic, has been reported useful in different domains such as awareness or wakefulness [38]. Clinical improvements were also showed thanks Zolpidem in VS [39]. Lamotrigine is an antiepileptic drug with mood-stabilizing properties. A recent case series showed lamotrigine's utility to encourage functional recovery particularly in patients spontaneously emerging from MCS. The antiglutamatergic effects may results in psychotropic effects and neuroprotective action [40]. The use of intrathecal Baclofen, used to treat spasticity, showed positive effects in improving level of consciousness in VS patients. Baclofen is a GABA-B receptor agonist; it probably modulates spinal cord segmental activities and the centripetal neuronal outputs reaching the cortex; it could also deregulate sleep-wake cycles interfering with consciousness. A direct brain effect is not possible because intrathecal administration leads to very low plasma levels of baclofen [41]. Wakefulness is regulated by the ascending arousal system that has two major branches. One arising from monoaminergic nuclei (the tuberomammillary nucleus containing histamine, the A10 cell group containing dopamine, the dorsal and median raphe nuclei containing serotonin and the locus coeruleus containing noradrenaline). The "flip-flop" switch model describes the mutual inhibition between the activating monoaminergic cells groups and the inhibiting ventrolateral pre-optic nucleus (VLPO, rich in GABA). During wakefulness, the monoaminergic groups prevail and inhibit VLPO. Antidepressant influence noradrenergic and serotoninergic systems subsequently promote the ascending arousal pathways [42-47].

Aim of this study was to evaluate the correlation between clinical examination and neuroradiological imaging in patients with DOC, for predicting the likely outcome of one's current standing.

\section{Materials and Methods}

\section{Study population}

Six patients ( 4 men and 2 female) were recruited. The patients (middle age of 54 years) suffered of DOC post-coma, outcome of severe acquired brain injuries (cerebral hemorrhage, cerebral ischemia, anoxia by cardiac arrest). The coma periods ranging from 21 days to 38 days (with an average of 30,8 days) (Table 1).

All the patients were examined in acute phase with brain (scan) CAT (computerized axial tomography) and/or MRI (magnetic resonance imaging). After, they were evaluated with electroencephalogram and fMRI. Repeated clinical assessments were conducted with neurological examination and rating scales such as Glasgow Coma Scale (GCS) and Disability Rating Scale (DRS) at the beginning (T1), middle (T2) and end (T3) of hospitalization.

The inclusion criteria of the study were:

1) SV o SMC;

2) Onset by not more than 20 months;

3) Absence of significant organic diseases related to the nervous system earlier to GCA;

4) Absence of neoplastic diseases in progress and of higher risk of epileptic status.

For clinical efficacy evaluation, we used neurological examination and clinical rating scales of the level of consciousness and disability: Disability Rating Scale (DRS), Rapport Coma/Near-Coma Scale (CNC),

\begin{tabular}{|c|c|c|c|c|c|}
\hline Patient & Age & Sex & Disease onset (months) & Etiology of Vegetative state \\
\hline C.S. & 34 years & M & 20 & Cerebral TC: Post-anoxic event (cardiac arrest) \\
\hline C.A. & 78 years & F & 19 & Cerebral TC: Severe ischemic lesions \\
\hline M.V. & 75 years & F & 19 & Right severe brain hemorrhage \\
\hline C.D. & 77 years & M & 20 & Cerebral TC: bilateral (right side>left side) frontal brain hemorrhage \\
\hline L.C. & 62 years & M & 20 & Cerebral TC: Right cerebellar-temporo-occipital sub-thalamic midbrain-pontine ischemia \\
\hline C.F. & 39 years & M & 19 & $\begin{array}{c}\text { Cerebral TC: cerebral hemorrhage due to ruptured arteriovenous malformation in the right } \\
\text { temporo-parietal-occipital region, left parasagittal region and corpus callosum (posterior third) }\end{array}$ & 31 days \\
\hline
\end{tabular}

Table 1: Clinical and demographic informations of patients. 
Glasgow Coma Scale (GCS) and Levels of Cognitive Functioning (LCF). A study was also performed with fMRI using visual stimulations (black and white checkerboard in motion), acoustic stimulation (random words and family's message, lasting thirty seconds each) and tactilesensory stimulation (conducted with the aid of a needle).

\section{Study treatments}

In all patients rehabilitative treatment was performed consisting of physiokinesitherapy (FKT) on a daily basis.

They were also pharmacologically treated as follows:

Patient 1: Sodium valproate $1250 \mathrm{mg} / \mathrm{die} / \mathrm{PEG}$, Lioresal $25 \mathrm{mg} / \mathrm{die} /$ PEG,Piracetam 9 gr/die/e.v, L-Dopa 750 mg/die;

Patient 2, allergic to Piracetam: L-Dopa $500 \mathrm{mg} / \mathrm{die} / \mathrm{PEG}$;

Patient 3: L-Dopa 750 mg/die, Levetiracetam 1000 mg/die/PEG;

Patient 4: L-Dopa 500 mg/die/PEG

Patient 5: L-Dopa 500 mg/die/PEG; Carbamazepine 600 mg/die;

Patient 6: L-Dopa $500 \mathrm{mg} /$ die/PEG

All patients were on medical supportive therapy (rehydration, nutritional calorie, vitamin, etc.) and prevention (preventive and curative therapy) and treatment of complications.

\section{Results}

\section{Patient 1}

C.S., M, 34 y, S.V. post- anoxic from 20 months result of cardiac arrest, severe and diffuse cortico-subcortical suffering ( 35 days of coma). At the time of hospitalization (T0): patient with tracheostomy and spontaneous breathing, apparent complete absence of environmental contact, eye spontaneous opening, with normal shape pupils but with no mild light response (left more right side); reaction to intense nociceptive stimulation with grimaces of the face and with flexion in decortication of the upper limbs. Flaccid paralysis was present, atavistic reflexes were present: snout, glabellar, "doll's eyes", grasping bilaterally, palm-chin bilaterally, Hoffmann bilaterally; widespread muscle hypertonicity with tendency of the elbow and wrist flexion bilaterally, Babinski with tendency to triple flexion bilaterally, occasional vegetative crisis (tachycardia, hypertension, sweating, etc.), previous seizures. Showy "alert" reactions to the intense acoustic stimulus with "timelapse/temporal extinction" phenomenon to repetition of the same, accompanied by reference to the gaze deviation toward the acoustic source. There were upper limbs' osteotendinous hyperreflexia, areflexia in the lower limbs. Patient with nasogastric tube and indwelling catheter, vegetative state.

Cerebral CT examination: Diffuse hyper-intense signal in the white matter in the DWI sequences. T1 weighted sequence with contrast, impregnation of cortical gray matter with rail aspect, of caudate nucleus and of putamen. Spectroscopic examination conducted at the level of the left semi-oval center: clear the peak lactate, index of anaerobic metabolism.

For the clinical evaluations have been used the following evaluation scales:

DRS: 25

GCS: 7

CNC: 4

\section{LCF: 1}

At T1: fMRI (tactile and dolorific stimulation): left temporal activation, right cerebellar hemispheric activation and right rolandic activation left posterior temporal activation. The functional examination of the first patient did not provide significant results to the acoustic stimulation such as music tracksor listening to words of strangers:

- listening to music: minimal bilateral temporal activation area;

- listening to recorded messages with the voices of the patient's family: bilateral widespread activation in temporal and polar regions and in posterior temporal regions, especially on the right and retrorolandic parietal right activation.

A minimal activation there was by listening to familiar voices through recorded messages.

fMRI study showed the presence of cortical areas responsive to the type of stimulation coherently in right occipital and frontal regions.

Listening to words (auditory task) is associated with activation of the cortex of bilateral temporal-island (more evident on the left) and frontal regions.

There is activation of the mesial occipital cortex as well as in the prefrontal white matter.

During visual task: significant activation of the cortex of the occipital lobes bilaterally.

At T2: patient began to turn with the head and conjugated gaze (eyes) toward the voice source, if called by name, only occasionally. For other functions he was stationary. For clinical assessments we used the following rating scales:

DRS: 23

GCS: 9

CNC: 2.9

LCF: 2

\section{Patient 2}

C.A., F, 78 years, S.V. by severe ischemic lesions: right insulotemporo-parietal-occipital and left periventricular (21 days of coma). At the time of hospitalization (T0): apparent complete absence of environmental contact, spontaneous eye opening, widespread muscle atrophy and hypotonia, ubiquitous deep areflexia, no response to the nociceptive stimulation, bilateral Babinski; atavistic signs: bilateral snout, palm-chin, glabellar, bilateral Hoffmann.

Examination cerebral CT

For clinical assessments we used the following rating scales:

DRS: 28

GCS: 3

CNC: 3

LCF: 1

At T1: fMRI (tactile-dolorific stimulation): bilateral perirolandic activation, increased on the right, right capsular and temporal activation. The functional analysis showed activation in incongruous areas with the type of stimulus presented (visual, tactile). Visual task: prevalent activation of the left temporal region. 
Listening to words: the prevalent activation is in the right temporoinsular region.

Listening to music: minimum activation in the left posterior temporal region.

Listening to recorded messages of familiar voice: activation in the left temporal, occipital and perirolandic areas, mesial occipital region.

At T2: patient did not change his clinical situation of vegetative state.

For clinical assessments we used the following rating scales:

DRS: 21

GCS: 11

CNC: 2

LCF: 2

\section{Patient 3}

M.V., F, 75 years, S.V., outcome of right severe brain hemorrhage treated surgically (32 days of coma). At the time of hospitalization (T0): apparent complete absence of environmental contact, absence of reaction to nociceptive stimulation, spontaneous tendency to posture in decortication (bending of the elbows and wrists), previous seizures, diffuse muscle hypertonia, bilateral silent hallux during the skin-plantar stimulation test; atavistic signs: snout, glabellar, palm-chin bilaterally. The patient was constantly with closed eyes and opposed resistance to passive opening of the eyelid.

For clinical assessments we used the following rating scales:

DRS: 29

GCS: 4

CNC: 3

LCF: 3

At T1: fMRI (tactile-dolorific stimulation): activation in left parietal perirolandic region, right cerebellar hemispheric and frontal interhemispheric regions, compatible with neuronal activation associated with stimulation, also smaller areas with similar characteristics in the left temporal and bilateral occipital regions.

The functional examination of the third patient showed activation compatible and congruous to the stimulation:

- listening to words: activation of temporoinsular regions especially on the left, and bilateral frontal, mesial-occipital and prefrontal white matter;

At T2: The patient began to open spontaneously eyes for periods of time more and more prolonged and, subsequently, to respond in a correct way congruent to simple questions, gesturing and trying to vocalize (with attempts to vocalization). The patient began environmental contact and, although very slow, interacts correctly through verbal and nonverbal language.

For clinical assessments we used the following rating scales:

DRS: 20

GCS: 12

CNC: 1

\section{LCF: 4}

\section{Patient 4}

C.D., M, 77 years, S.V., outcome of bilateral (right side>left side) frontal brain hemorrhage ( 28 days of coma).

At the time of hospitalization (T0): alert patient, without deficits of the cranial nerves (as estimated), with eye spontaneous opening and closing; nociceptive stimulation caused the right upper limb flexion in decortication, while algic stimulation of the face induced facial grimaces. Patient with nasogastric tube (NGT), central venous catheter (CVC), spontaneously breathing with cuffed tracheostomy tube, urinary catheter. He presented atavistic reflexes. Vegetative state.

Examination cerebral CT: Hemorrhagic lesion in right basal nuclei, tetra-ventricular flood (surgically evacuated and subsequent placement of ventricular-peritoneal shunt), pneumatization of subarachnoid space (air-fluid levels in polar front region).

For clinical assessments we used the following rating scales:

DRS: 26

GCS: 6

CNC: 4

LCF: 1

At T1:

- Listening to words: minimum activation in the left posterior temporal and occipital region.

- Listening to music interpreted by the patient: no significant activation.

- Listening to music: minimum activation of bilateral temporal area.

- Listening to recorded messages with the patient's familiar voices: widespread activation in bilateral temporo-polar and posterior temporal regions, especially on the right and right parietal retrorolandic region.

\section{At T2: death.}

\section{Patient 5}

L.C., M, 62 years, S.V., outcome of right cerebellar-temporooccipital sub-thalamic midbrain-pontine ischemia (31 days of coma).

At the time of hospitalization (T0): the patient is alert, in vegetative state. To the nociceptive stimulation patient responded with some changes in facial expression. Snout reflex was present, the winking was absent. The osteotendinous reflexes were less elicited at lower limbs, which appeared hypotonic; instead they were hyperkinetic at upper limbs, which occurred hypertonic. Wrist dorsiflexion evoked clonus bilaterally exhaustible. To plantar cutaneous stimulation, the big toe was bilaterally dumb. Patient with central venous catheter (CVC) in the right subclavian artery, indwelling catheter, spontaneously breathing through a tracheostomy tube. There were bilateral calcaneal bedsores (heel). Also it was present stereotypical movements of chewing, yawning, snout reflex, palm-chin and grasping reflexes.

Examination cerebral CT: Large ischemic lesion in the right cerebellar hemisphere, in the right temporo-occipital area, and an involvement of bridge and central midbrain and diencephalon (thalamus and sub thalamus).

For clinical assessments we used the following rating scales: 
DRS: 25

GCS: 8

CNC: 4

LCF: 1

At T1

Visual task: prevailing activation of left temporal region.

- Listening to words: prevailing activation in the right temporoinsular region. Interesting left thalamic location in the iuxtaventricolar region.

- Listening to music: minimum activation in the left posterior temporal region. Not significant.

- Listening to recorded messages of familiar voice: activation in the temporal area left occipital and peri-rolandic region, mesialoccipital area.

- During the left forearm sensory stimulation: activation of the temporal and occipital cortex on the left.

- The functional study was characterized by weak or incongruent or typical according to the stimulus activations, that was limited only to the primary areas, without involvement of specific associative areas.

- Listening to recorded messages by family voice was associated with cortical activation of the temporal, occipital and perirolandic regions on the left, although the encephalic trunk evoked potentials were absent in the BAEP.

Important was the activation in the mesial occipital and minimally in the basal forebrain regions.

\section{At T2: stationary}

For clinical assessments we used the following rating scales:

IDRS: $25 \mathrm{mg}$

GCS: 8

CNC: 4

LCF: 1

\section{Patient 6}

C.F., M, 39 years, S.V., outcome of cerebral hemorrhage due to ruptured arteriovenous malformation in the right temporo-parietaloccipital region, left parasagittal region and corpus callosum (posterior third) (38 days coma)

At the time of hospitalization (T0): minimal responsiveness state, with minimal reactions aimed at right upper limb in response to painful or intense vocal stimuli. Spastic quadriplegia was present, with attitude in flexion of the upper limbs and extension of the lower limbs. The tendon reflexes were less evoked at four limbs. It was also present the glabellar reflex, palm chin reflex, the snout reflex. To deep cutaneous stimulation, the big toe remained silent bilaterally. The equinovarus feet were bilaterally.

\section{Examination cerebral CT}

T2-weighted sequences, extended hemorrhagic outcome in the right parietal region and in corpus callosum (splenium).
For clinical assessments we used the following rating scales:

DRS: 22

GCS: 7

CNC: 3

LCF: 2

At T1:

- Visual task: significant activation in bilateral occipital cortex.

- The fMRI examination showed a proper activation of occipital areas delegated to the analysis of the visual stimulus.

At T2: exitus.

\section{Discussion}

Clinical evaluation performed with rating scales of consciousness and disability degree, showed in all patients an increase of absolute value of GCS and LCF and a reduction of DRS and CNC. In particular, for the patient 3 we observed an important increase of GCS score (from 4, T0 to 12, T2) and LCF score (from 3 at T0 to 4 at T2) and an important decrease of CNC score (from 3 at T0 to 1 at T2) and DRS score (from 29 at $\mathrm{T} 0$ to 20 at T2).

We obtained most interesting observations concerning the relationship between the prognosis and patient characteristics with functional MRI (Figures 1-4).

The study performed with sensory pain stimulation at upper right distal limb in patient 1 showed circumscribed signal alterations in the left temporal cortex and in the right cerebellar hemispheric and rolandic regions.

The cognitive stimulation paradigm (listening to familiar voices and tunes) elicited in patient 1 cortical activation in the left posterior temporal and right frontal regions.

The functional MRI of patient 2, showed bilateral perirolandic activation (more to the right) and right capsular and temporal activation. Also in this patient listening to familiar voices caused a significant activation.

Functional MRI of the patient 3, always performed with sensorydolorific stimulation of right upper, showed the presence of the signal alteration areas in BOLD sequence in the left parietal perirolandic region, right cerebellar hemispheric and frontal interhemispheric regions, compatible with neuronal activation associated to stimulation. In patient 3, we reported weak activation extended to higher order processing areas as in the case of verbal stimulation and the tactiledolorific stimulation which led to an activation of the mesial posterior cortex, the first and the mesial anterior cortex, the second (atypical activation). This is important in light of the role played by the mesial cortex in interpretation of self-related stimuli. This implies that the patient may have maintained a minimum self-consciousness.

The most significant stimulations were those verbal to emotional content (familiar voices), even if in the patient 4 the stimulations induced activation despite a context of generalized functional silence.

In patients number 1 and 5 the verbal message induced the typical activation, plus a signal at the level of the mesial occipital cortex.

The patient 4 and 6 prognosis was unfortunate because they 
Citation: Sabino D, Riccardo M, Maria D, Alessia GG, Andrea S, et al. (2017) Prognostic and Diagnostic Value of Clinical Examination and fMRI in the Evaluation of Patients in a Vegetative State. J Neurol Neurophysiol 8: 430. doi:10.4172/2155-9562.1000430
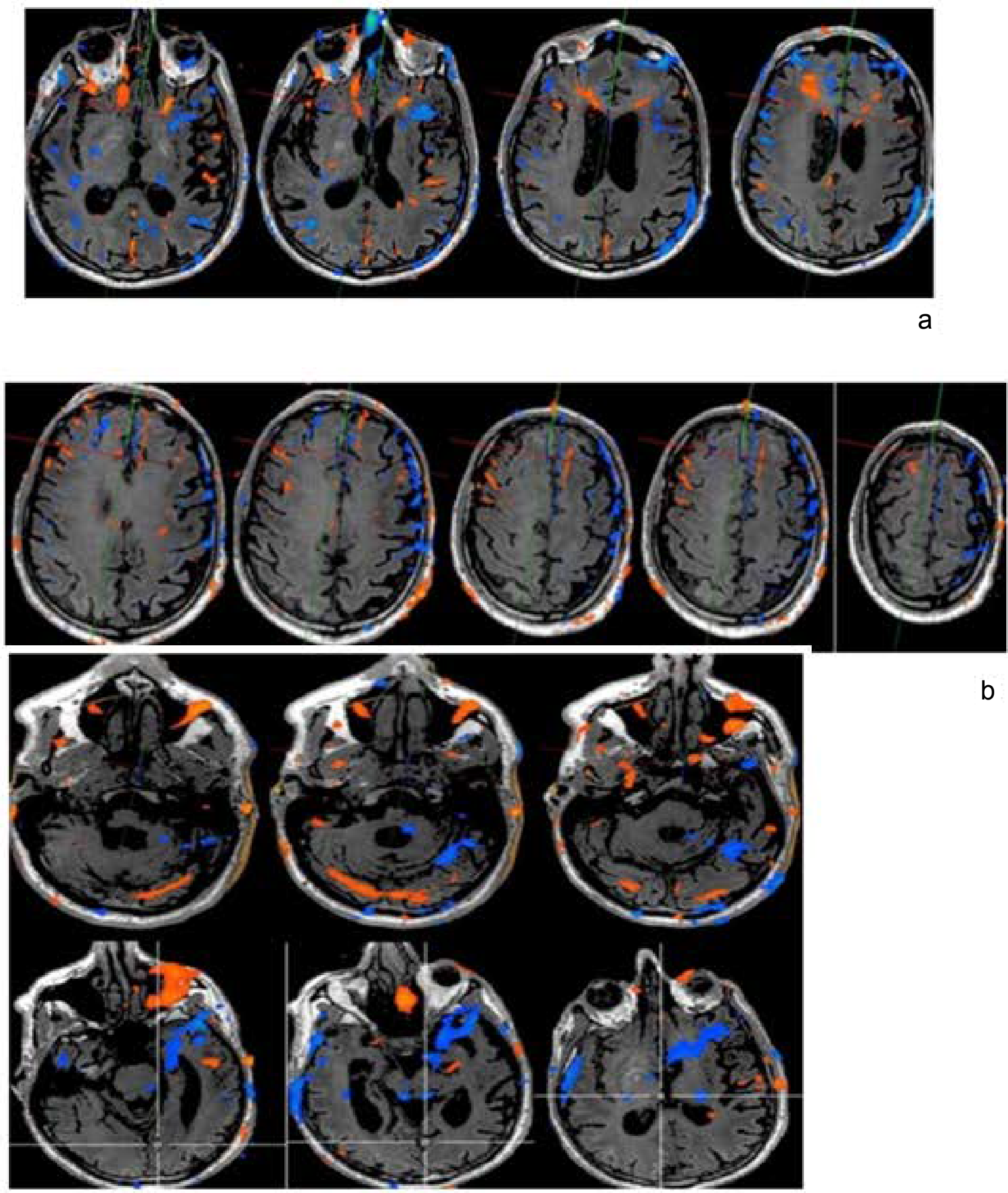

b

C

Figure 1: a: Listening to words (auditory task) is associated with activation of the cortex of bilateral temporal-island (more evident on the left) and frontal regions; there is an activation of the mesial occipital cortex as well as in the prefrontal white matter; $\mathbf{b}$ : Tactile and dolorific stimulation of left palm hand: right rolandic and pre-rolandic cortex activation, frontal interhemispheric activation; c: Visual task: significant activation of the cortex of the occipital lobes bilaterally. 
Citation: Sabino D, Riccardo M, Maria D, Alessia GG, Andrea S, et al. (2017) Prognostic and Diagnostic Value of Clinical Examination and fMRI in the Evaluation of Patients in a Vegetative State. J Neurol Neurophysiol 8: 430. doi:10.4172/2155-9562.1000430

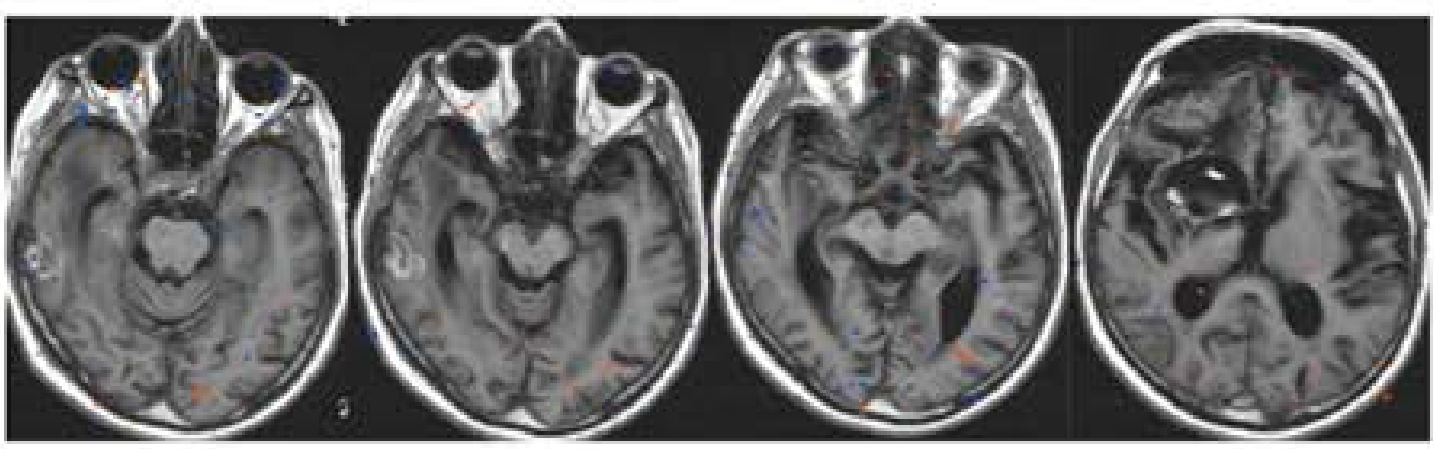

a
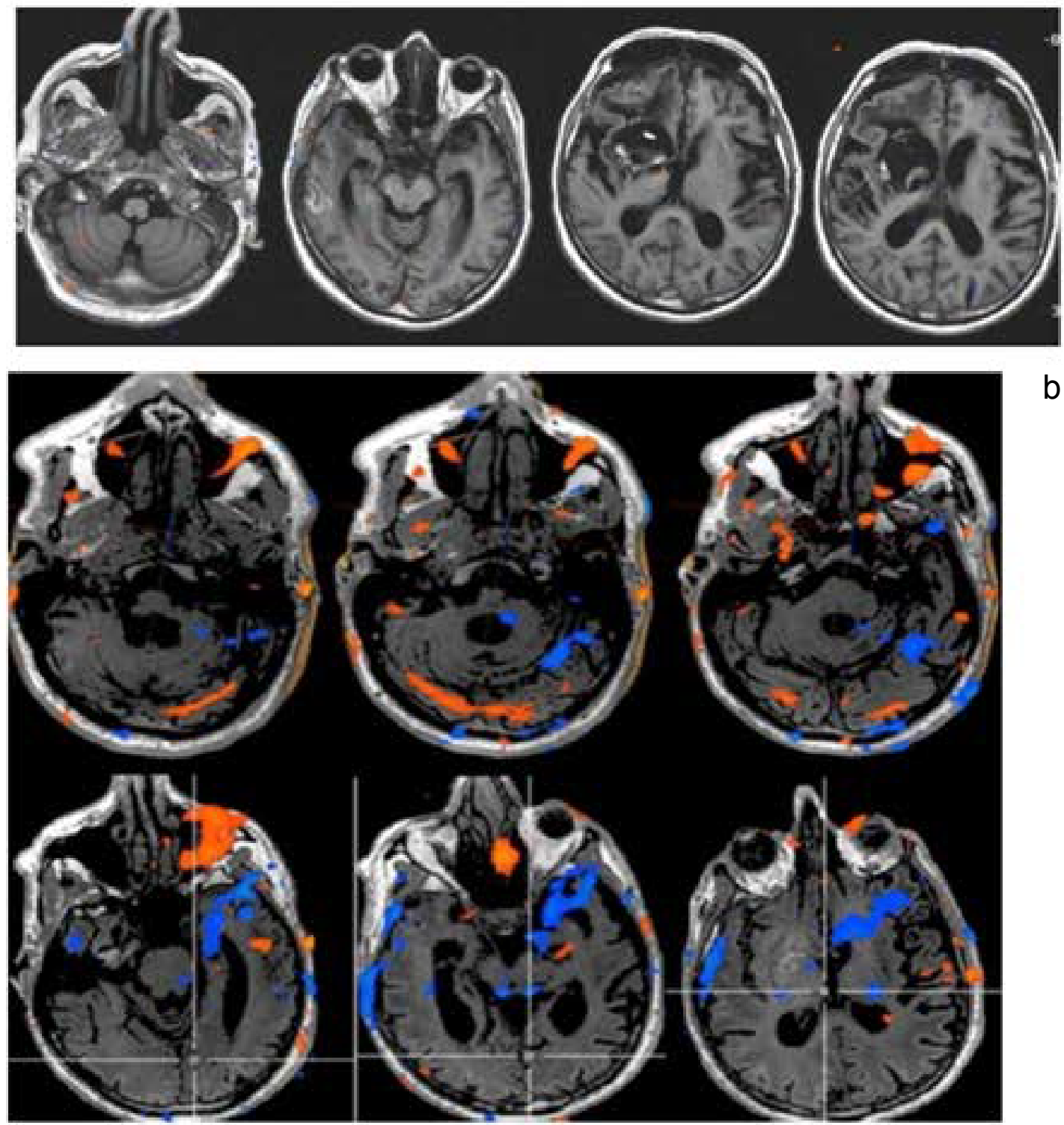

C

Figure 2: a: Listening to words: minimum activation in the left posterior temporal and occipital region; $\mathbf{b}$ : Listening to music interpreted by the patient: no significant activation; c: Listening to music: minimum activation of bilateral temporal area. 
Citation: Sabino D, Riccardo M, Maria D, Alessia GG, Andrea S, et al. (2017) Prognostic and Diagnostic Value of Clinical Examination and fMRI in the Evaluation of Patients in a Vegetative State. J Neurol Neurophysiol 8: 430. doi:10.4172/2155-9562.1000430
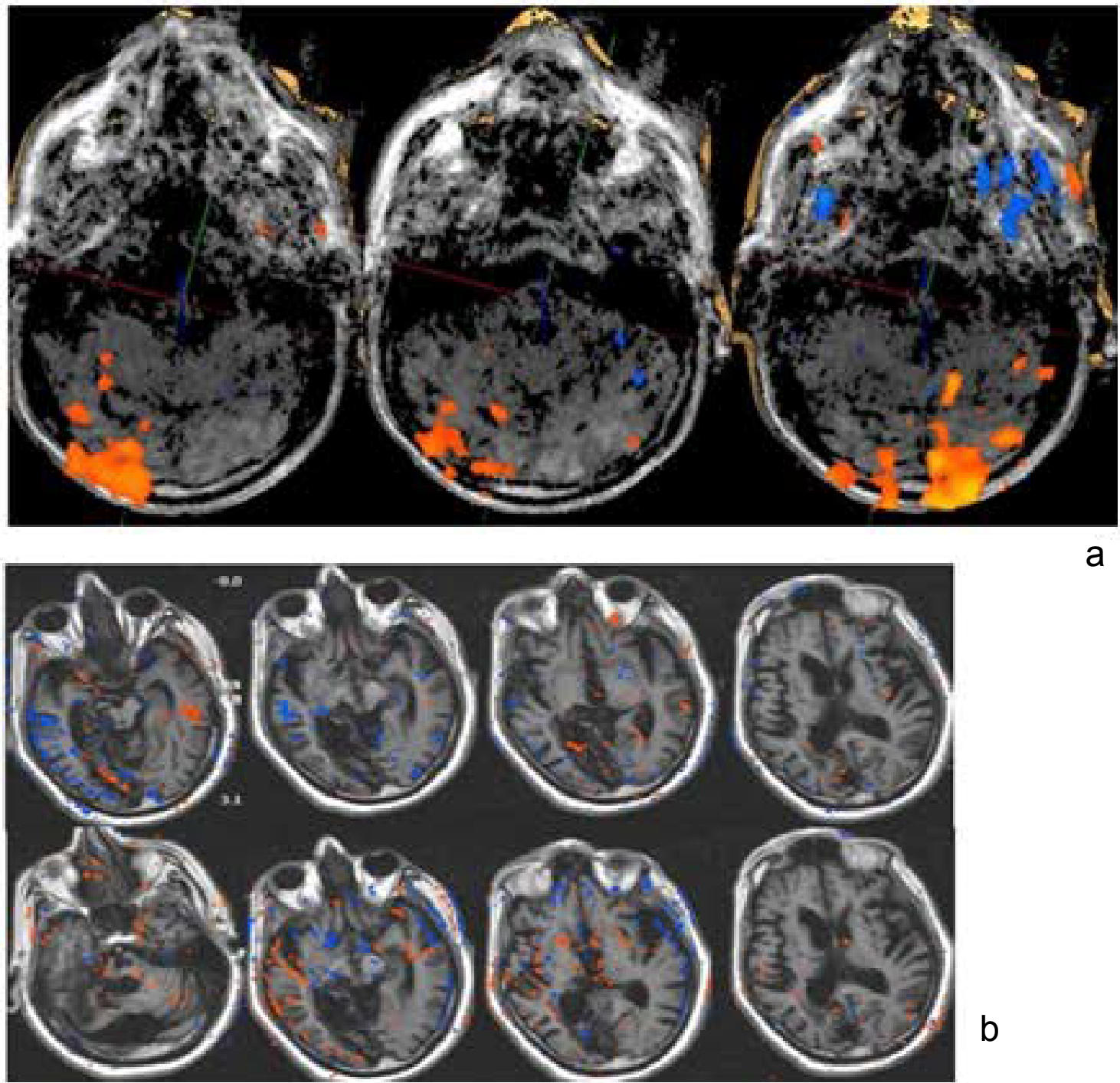

a

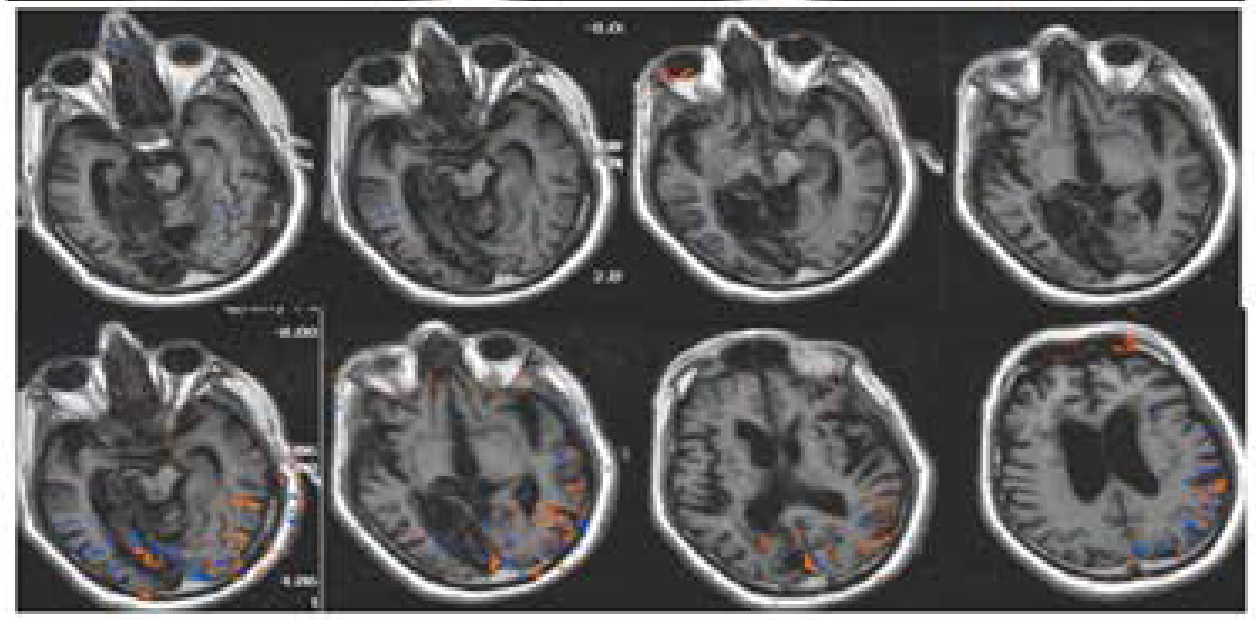

b

Figure 3: a: Listening to recorded messages by family voice was associated with cortical activation of the temporal, occipital and perirolandic regions on the left, although the encephalic trunk evoked potentials were absent in the BAEP. Important was the activation in the mesial occipital and minimally in the basal forebrain regions; b: Listening to words: prevalent activation in the right temporo-insular region. Interesting left thalamic location in the iuxtaventricolar region; c: Listening to recorded messages of familiar voice: activation in the temporal area, left occipital and peri-rolandic region, mesial-occipital area. 


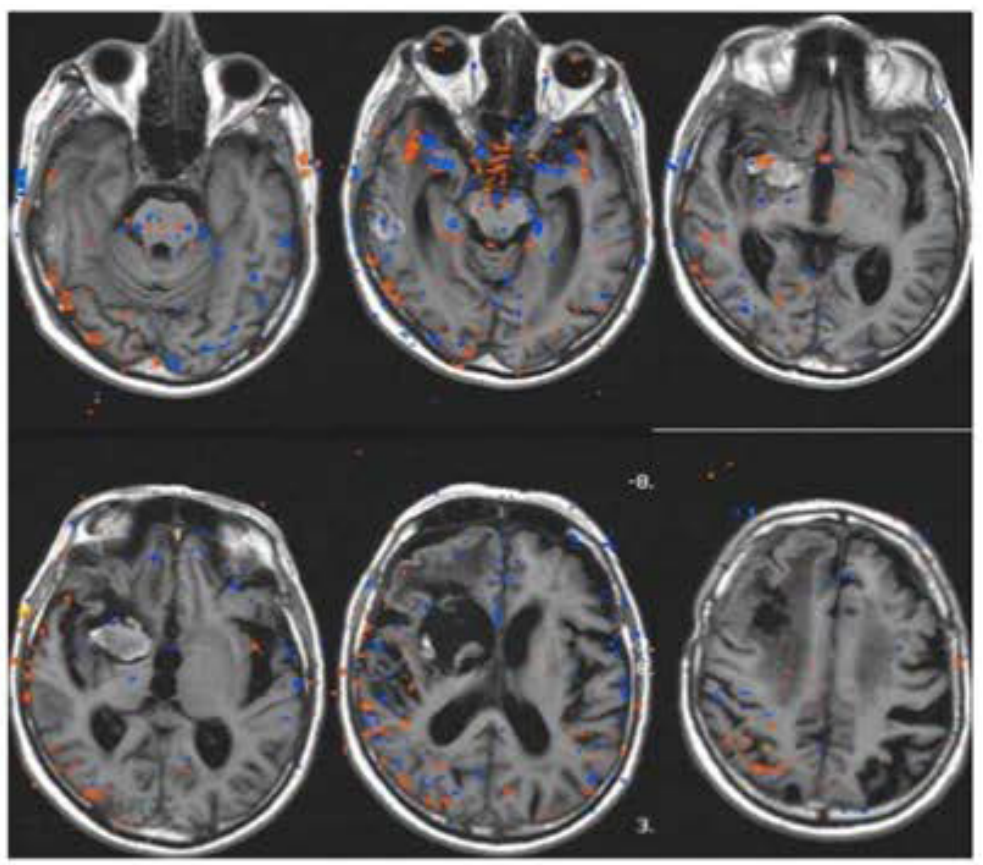

Figure 4: Visual task: Significant activation in bilateral occipital cortex.

died, while patients 2 and 5 registered improvements but still are in a vegetative state. Probably the hemorrhagic event (DAI) in patients 4 and 6 was behind the worst outcome.

Many of the factors described previously had weight in directing determined a worse prognosis. First of all there is the vascular etiology (not from cranioencephalic trauma for all cases).

We saw that the prognosis was exitus in more than half of the cases (53\%), the vegetative state in a third of cases $(32 \%)$. Each patient then presented suggestive elements for an unfavorable course.

In patient 4 , advanced age and posture in decortication in history supported an unfavorable outcome. The most important was fMRI that showed a state of almost functional silence; brain activation was minimal or absent, whether to the acoustic stimulus and to verbal and musical stimulus. The relatives voices in the form of recorded messages evoked a weak response at the level of the right temporal cortex and right perirolandic cortex.

The patient 5 survived in SV. Older age and a high lesion load, for the most engaging areas of extreme importance such as the brain stem and midbrain, were suggestive of an extremely poor outcome. The unilateral of the seat of pontine and midbrain lesions, is probably the aspect that has saved the patient's life.

Data obtained from the fMRI showed mostly the incongruity of the areas activated by the task than expected. Infact, visual stimulation has determined an activation of the left temporal cortex rather than the bilateral occipital cortex, where listening to words produced an activation of right temporal cortex (as the previous patient) and insular bilateral activation instead of classical localization temporoparietal left in Wernicke area. Listening to music has not elicited any cortical reaction.

Also the left forearm stimulation, which normally would cause an activation of the somatosensory area of the contralateral parietal lobe, induced a weak activation in the occipital and temporal regions, for more ipsilateral. These abnormalities in the findings can be attributed to anatomical and functional impairment of the nervous signal transmission, to recruitment of non-injured areas and functionally related, to uncontrolled divergence of signal for loss of inhibitory mechanisms during selective attention. Listening to family messages induced a weak activation of the temporal, occipital, perirolandic areas on the left, and the medial cortex of the wedge.

Patient 1 suffered a severe anoxic damage. At the time of hospitalization, he had a number of aspects related to an unfavorable prognosis such as the nature of the cerebral insult and the attitude in decortication. The EEG showed theta-delta activity of ultra-low voltage, not reactive. The MRI showed a severe global impairment of cortical gray matter and substance subcortical such as white matter. However it is the only patient who to $\mathrm{MRI}$ showed consistent activation coherent with the type of administered stimulus.

In fact, the visual stimulus induced activation of the occipital visual cortex; the verbal stimulus produced a signal at the level of the left temporal cortex. The touch-reliever stimulation of the left hand palm has produced an activation of controlateral rolandic and prerolandic laps. In all cases the different types of stimuli elicited a signal in the primary processing areas, be it visual or auditory or sensory. In addition, although weakly, other cortical areas were activated during the administration of the stimuli. In this case, listening to words also caused insula activation, in the medial occipital cortex and in the prefrontal white substance. To insular cortex (insular lobe) are attributed functions related to the sphere of emotions and homeostasis. These include the perception, motor control, self-awareness, cognitive functions, the interpersonal experience. In this case listening to words might have aroused a weak emotional tone of acoustic stimulus. Also, it is interesting the activation of medial cortex at the level of the wedge and precuneus (a phenomenon also observed in the patient 5). The posterior medial regions, including the posterior cingulate and the 
precuneus, appear to be involved in creating environment awareness, and its activity, in particular of the precuneus, seems to be correlated with the consciousness degree of the person.

However, these results refer to fMRI studies performed with the patient at rest and not during verbal stimulation.

Finally it is worth noting the activation pattern after tactile reliever stimulus that in addition to involving the primary somatosensory area extends slightly to the medial frontal cortex: this is involved in the selfrelated stimulation, also is a secondary processing area of reliever stimulus responsible of the emotional coloring of the latter. Therefore recruitment of medial cortex both front and back may be suggestive of conservation baseline mental activity, between qualitatively crude processing of sensory stimuli and interpretation with a minimal emotional imprint.

Patient 6 dead despite the young age, neurophysiological examinations (BAEP) encouraging and fMRI response coherent with stimulus type (occipital cortex activation to visual stimuli). Some prognostic factors in this direction would be the hemorrhagic nature of brain injuries, the load important lesion and the involvement of the body callosum at the body level and the splenius.

Patient 3 was the only one had positive outcome, with emergence from vegetative state and demonstration of ability to shoot, although inconsistently, head and conjugate gaze, toward the voice source if called by name. This is in line not only with a picture of atypical fMRI temporal (acoustic), parietal (tactile-dolorific) and occipital (visual) activation, but also with clinical evidence of "non-reflex reactions" to "alert "the intense acoustic stimulus.

\section{Conclusion}

Despite the small number of patients, our study, in accordance with literature, may confirm the central role of nature of brain injuries as prediction marker for the outcome (in our study the non-traumatic cerebral vascular injuries is the most serious, particularly hemorrhagic type) and fMRI direct visualization of the areas of cortical activation (thus providing information complementary to clinical assessment and helping monitoring treatment effects). So, we affirm:

a) The major meaning of clinical aspects compared to instrumental procedures for diagnosis and prognosis (importance of the clinical evaluation of "signs of non-reflex/non-conscious behavior");

b) The great importance of fMRI for diagnosis, prognosis and outcome in patients with DOC (atypical activation identified in patient 1 and 3 comes before the transition from V.S. to M.S.C.);

c) The significance of difference between the group V.S. and its subgroup V.S.+ (non-reflex/non-conscious behavior). This second subgroup of patients is characterized by nonconscious and non-automatic activities (patients 1 and 3 are associated with positive outcomes). In fact, the subgroup V.S.+ have a better prognosis than the group V.S. The pattern fMRI of subgroup V.S.+ shows modest atypical activation. In addition, those patients have a good response to L-Dopa and dopaminergic therapy (less effective in VS patients).

Our study relates diagnostic information in patients with DOC and confirms the literature data about the advantages of fMRI [48]. However, these data refers to a very small sample of patients (only 6 patients, two of whom died) and therefore are not statistically significant.

\section{Conflict of Interest}

Authors declare no potential sources of conflict of interest in manuscript (any interest or relationship, financial or otherwise that might be perceived as influencing an author's objectivity).

\section{References}

1. Jellinger KA (2009) Functional pathophysiology of consciousness. Neuropsychiatr 23: 115-133.

2. Tononi G, Edelman GM (1998) Consciousness and complexity. Science 282 1846-1851.

3. Plum F, Posner JB (2015) The diagnosis of stupor and coma. Brain Nerve 67 344-345.

4. Crick F, Koch C (2003) A framework for consciousness. Nat Neurosci 6: 119 126.

5. Boly M, Sasai S, Gosseries O, Oizumi M, Casali A, et al. (2015) Stimulus set meaningfulness and neurophysiological differentiation: A functional magnetic resonance imaging study. PLoS ONE 10: e0125337.

6. Tononi G (2012) Integrated information theory of consciousness: An updated account. Arch Ital Biol 150: 293-329.

7. Monsef Kasmaei V, Asadi P, Zohrevandi B, Raouf MT (2015) An epidemiologic study of traumatic brain injuries in emergency department. Emerg (Tehran) 3 : 141-145.

8. Björkman J, Hallikainen J, Olkkola KT, Silfvast T (2015) Epidemiology and aetiology of impaired level of consciousness in prehospital non-trauma patients in an urban setting. Eur J Emerg Med 23: 375-380.

9. Encyclopédie médico-chirurgicale I-26.495-A-10

10. Plum F, Posner J (1982) Dignostic de la stupeur et des comas. Paris, pp: 1-93

11. Multi-Society Task Force on PVS (1994) Medical aspects of the persistent vegetative state (1). N Engl J Med 330: 1499-1508.

12. Giacino JT, Ashwal S, Childs N, Cranford R, Jennett B, et al. (2002) The minimally conscious state: Definition and diagnostic criteria. Neurology 58 349-353.

13. Bruno MA, Vanhaudenhuyse A, Thibaut A, Moonen G, Laureys S (2011) From unresponsive wakefulness to minimally conscious PLUS and functional locked-in syndromes: Recent advances in our understanding of disorders of consciousness. J Neurol 258: 1373-1384.

14. Majerus S, Gill-Thwaites H, Andrews K, Laureys S (2005) Behavioral evaluation of consciousness in severe brain damage. Prog Brain Res 150: 397-413.

15. Gill-Thwaites H (2006) Lotteries, loopholes and luck: Misdiagnosis in the vegetative state patient. Brain Inj 20: 1321-1328.

16. Childs NL, Mercer WN, Childs HW (1993) Accuracy of diagnosis of persistent vegetative state. Neurology. 43: 1465-1467.

17. Andrews K, Murphy L, Munday R, Littlewood C (1996) Misdiagnosis of the vegetative state: retrospective study in a rehabilitation unit. BMJ 313: 13-16.

18. Andrews K (2004) Medical decision making in the vegetative state: Withdrawal of nutrition and hydration. NeuroRehabilitation 19: 299-304.

19. Laureys S, Faymonville ME, Peigneux P, Damas $P$, Lambermont $B$, et al (2002) Cortical processing of noxious somatosensory stimuli in the persistent vegetative state. Neuroimage 17: 732-741.

20. Boly M, Faymonville ME, Schnakers C, Peigneux P, Lambermont B, et al. (2008) Perception of pain in the minimally conscious state with PET activation An observational study. Lancet Neurol 7: 1013-1020.

21. Schnakers C, Vanhaudenhuyse A, Giacino J, Ventura M, Boly M, et al. (2009) Diagnostic accuracy of the vegetative and minimally conscious state: Clinical consensus versus standardized neurobehavioral assessment. BMC Neurol 9 : 35 .

22. Whyte J, Katz D, Long D, DiPasquale MC, Polansky M, et al. (2005) Predictors of outcome in prolonged posttraumatic disorders of consciousness and assessment of medication effects: A multicenter study. Arch Phys Med Rehabil 86: 453-462.

23. Lammi MH, Smith VH, Tate RL, Taylor CM (2005) The minimally conscious 
Citation: Sabino D, Riccardo M, Maria D, Alessia GG, Andrea S, et al. (2017) Prognostic and Diagnostic Value of Clinical Examination and fMRI in the Evaluation of Patients in a Vegetative State. J Neurol Neurophysiol 8: 430. doi:10.4172/2155-9562.1000430

state and recovery potential: A follow-up study 2 to 5 years after traumatic brain injury. Arch Phys Med Rehabil 86: 746-754.

24. Laureys S, Owen AM, Schiff ND (2004) Brain function in coma, vegetative state and related disorders. Lancet Neurol 3: 537-546.

25. Laureys S (2005) The neural correlate of (un)awareness: Lessons from the vegetative state. Trends Cogn Sci 9: 556-559.

26. Laureys S, Goldman S, Phillips C, Van Bogaert P, Aerts J, et al. (1999) Impaired effective cortical connectivity in vegetative state: Preliminary investigation using PET. Neuroimage 9: 377-382.

27. Gusnard DA, Raichle ME, Raichle ME (2001) Searching for a baseline: Functional imaging and the resting human brain. Nat Rev Neurosci 2: 685-694.

28. Maquet $P(2000)$ Functional neuroimaging of normal human sleep by positron emission tomography. J Sleep Res 9: 207-231.

29. Baars BJ, Ramsøy TZ, Laureys S (2003) Brain, conscious experience and the observing self. Trends Neurosci 26: 671-675.

30. Laufs H, Lengler U, Hamandi K, Kleinschmidt A, Krakow K (2006) Linking generalized spike-andwave discharges and resting state brain activity by using EEG/fMRI in a patient with absence seizures. Epilepsia 47: 444-448.

31. Soddu A, Boly M, Papa M, Laureys S, Malach R (2009) Reaching across the abyss: Recent advances in functional magnetic resonance imaging (fMRI) and their potential relevance to deficits of consciousness. In S Laureys, ND Schiff, AM Owen (Eds.), Coma science: Clinical and ethical implications. Prog Brain Res, p: 177.

32. Laureys S, Faymonville ME, Peigneux $P$, Damas $P$, Lambermont $B$, et al. (2002) Cortical processing of noxious somatosensory stimuli in the persistent vegetative state. Neuroimage 17: 732-741.

33. Boly M, Faymonville ME, Peigneux P, Lambermont B, Damas P, et al. (2004) Auditory processing in severely brain injured patients: Differences between the minimally conscious state and the persistent vegetative state. Arch Neurol 61 : 233-238.

34. Giacino JT, Hirsch J, Schiff N, Laureys S (2006) Functional neuroimaging applications for assessment and rehabilitation planning in patients with disorders of consciousness. Arch Phys Med Rehabil 87: S67-76.

35. Di H, Boly M, Weng X, Ledoux D, Laureys S (2008) Neuroimaging activation studies in the vegetative state: Predictors of recovery? Clin Med 8: 502-507.

36. Georgiopoulos M, Katsakiori P, Kefalopoulou Z, Ellul J, Chroni E, et al. (2010) Vegetative state and minimally conscious state: A review of the therapeutic interventions. Stereotact Funct Neurosurg 88: 199-207.
37. Meythaler JM, Depalma L, Devivo MJ, Guin-Renfroe S, Novack TA (2001) Sertraline to improve arousal and alertness in severe traumatic brain injury secondary to motor vehicle crashes. Brain Inj 15: 321-331.

38. Whyte J, Hart T, Vaccaro M, Grieb-Neff P, Risser A, et al. (2004) Effects of methylphenidate on attention deficits after traumatic brain injury: $A$ multidimensional, randomized, controlled trial. Am J Phys Med Rehabil 83: 401-420.

39. White J, Myers R (2009) Incidence of clinically significant responses to zolpidem among patients with disorders of consciousness: A preliminary placebo controlled trials. Am J Phys Med Rehabil 88: 410-418.

40. Showalter PE, Kimmel DN (2000) Stimulating consciousness and cognition following severe brain injury: A new potential clinical use for lamotrigine. Brain Inj 14: 997-1001.

41. Sarà M, Pistoia F, Mura E, Onorati P, Govoni S (2009) Intrathecal baclofen in patients with persistent vegetative state: 2 hypotheses. Arch Phys Med Rehabil 90: 1245-1249.

42. Pistoia F, Mura E, Govoni S, Fini M, Sarà M (2010) Awakenings and awareness recovery in disorders of consciousness: Is there a role for drugs? CNS Drugs 24: 625-638.

43. Bagnato S, Boccagni C, Sant'angelo A, Prestandrea C, Rizzo S, et al. (2012) Patients in a vegetative state following traumatic brain injury display a reduced intracortical modulation. Clin Neurophysiol 123: 1937-1941.

44. Lapitskaya N, Moerk SK, Gosseries O, Nielsen JF, de Noordhout AM (2012) Corticospinal excitability in patients with anoxic, traumatic and non-traumatic diffuse brain injury. Brain Stimul 6: 130-137.

45. Fritsch B, Reis J, Martinowich K, Schambra HM, Ji Y, et al. (2010) Direct current stimulation promotes BDNF-dependent synaptic plasticity: Potential implications for motor learning. Neuron 66: 198-204.

46. Paquette C, Sidel M, Radinska BA, Soucy JP, Thiel A (2011) Bilatera transcranial direct current stimulation modulates activation-induced regional blood flow changes during volontary movement. J Cereb Blood Flow Metab 31: 2086-2095.

47. Bowden MG, Woodbury ML, Duncan PW (2013) Promoting neuroplasticity and recovery after stroke: Future directions for rehabilitation clinical trials. Curr Opin Neurol 26: 37-42.

48. De Salvo S, Caminiti F, Bonanno L, De Cola MC, Corallo F, et al. (2015) Neurophysiological assessment for evaluating residual cognition in vegetative and minimally conscious state patients: A pilot study. Funct Neurol 30: 237-244 